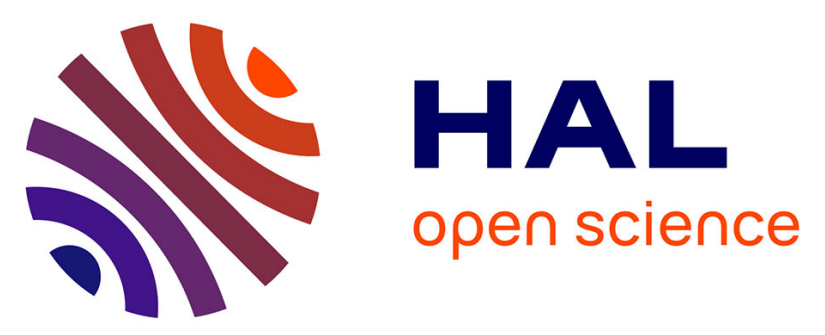

\title{
TROP-14 - Attitudes et pratiques vis à vis du paludisme dans une communauté amérindienne de Guyane
}

\author{
E. Mosnier, Maylis Douine, L. Musset, S. Rhodes, O. Gaudin, Damien Davy, \\ Loïc Epelboin, P. Pieter, R. Mutricy, Félix Djossou
}

\section{- To cite this version:}

E. Mosnier, Maylis Douine, L. Musset, S. Rhodes, O. Gaudin, et al.. TROP-14 - Attitudes et pratiques vis à vis du paludisme dans une communauté amérindienne de Guyane. 17es Journées Nationales d'Infectiologie, Jun 2016, Lille, France. Médecine et maladies infectieuses 46 (2016) 107-108, 46 (4), pp.107 - 108. inserm-01410981

\section{HAL Id: inserm-01410981 https://www.hal.inserm.fr/inserm-01410981}

Submitted on 6 Dec 2016

HAL is a multi-disciplinary open access archive for the deposit and dissemination of scientific research documents, whether they are published or not. The documents may come from teaching and research institutions in France or abroad, or from public or private research centers.
L'archive ouverte pluridisciplinaire HAL, est destinée au dépôt et à la diffusion de documents scientifiques de niveau recherche, publiés ou non, émanant des établissements d'enseignement et de recherche français ou étrangers, des laboratoires publics ou privés. 


\section{TROP-12}

Spécificités clinico-biologiques comparées à la dengue d'une arbovirose guyanaise méconnue : le virus tonate

R. Mutricy (1), L. Epelboin (1), E. Martinez-Lorenzi (2), E. Calciatti (3), S. Matheus (4), D. Blanchet (1), F. Djossou (1), D. Rousset (4)

(1) $\mathrm{CH}$ de Cayenne,

(2) Centre Médical Interarmées, Cayenne,

(3) CH Ouest Guyanais, Saint Laurent du Maroni,

(4) Institut Pasteur de Guyane.

Introduction Le virus Tonate (TONV) est un arbovirus découvert en 1973 en Guyane française, qui appartient à la famille des Togaviridae, genre Alphavirus. Il s'agit du sous-type IIIb du complexe des virus de l'encéphalite équine vénézuélienne. Les publications sont rares : une étude séroprévalence a montré que $10 \%$ de la population guyanaise était séropositive à ce virus, et un cas clinique d'encéphalite mortelle a été rapporté. Notre objectif était de décrire les caractéristiques de l'infection par cet arbovirus.

Matériels et méthodes Étude rétrospective multicentrique incluant les patients avec infection par le virus Tonate pris en charge dans les hôpitaux et centres de santé de Guyane de 2003 à 2014. Les IgM TONV étaient réalisées conjointement aux demandes de sérologie arbovirus. Tous les patients avec IgM TONV positives ont été réévalués et classés en probabilité forte (tableau clinique typique d'arbovirose et séroconversion IgM), moyenne (tableau clinique typique et IgM positives), faible (tableau clinique atypique et IgM positives) et exclue (dossier absent, diagnostic alternatif certain, tableau clinique non compatible avec une arbovirose ou IgM Tonate positifs connus antérieurement). Les cas ont été comparés avec un groupe témoin de patients avec dengue Ag NS1+ (2 témoins /cas) appariés sur le sexe et l'âge.

Résultats Sur 12 ans, 203 patients ont présenté des IgM TONV positives dont 170 ont été exclus $(67=$ dossier absent, $103=$ autre diagnostic (dont paludisme 29 ; dengue 11 ; chikungunya 10 ; fièvre Q 5 ; infectieux avec agent identifié 24 , infectieux avec agent non identifié 7 , cause non infectieuse 17). Trente-trois cas ont été inclus ( 3 de probabilité forte, 17 moyenne et 13 faibles). Seize (48.5\%) étaient des hommes, médiane d'âge : 35 ans, (IQ [22 - 47]). En analyse bivariée, les variables significativement associées à l'infection par TONV vs. dengue étaient : toux $(9(27.3 \%)$ vs. $6(9.1 \%), \mathrm{p}=0.04)$, leucocytes $>10 \mathrm{G} / \mathrm{L}(6(18.2 \%)$ vs. $2(3 \%) \mathrm{p}=0.03)$. Les variables présentant une tendance vers l'association à l'infection par TONV vs. dengue étaient: lombalgies $(8(24.2 \%)$ vs, $6(9.1 \%)$, p $=0.053)$, hospitalisation $(10(30.3 \%)$ vs.10 $(15.2 \%), \mathrm{p}=0.08)$, anémie $(5(15 \%)$ vs. $2(3 \%) \mathrm{p}=0.052)$. En multivariée seul la toux était associée de façon indépendante à l'infection par TONV (OR 22.7; IC95\% 1.95-263.9). Aucune forme sévère ni aucun décès n'ont été rapportés.

Conclusion Le virus Tonate a une présentation clinico-biologique bénigne, peu spécifique et difficile à différencier de la dengue classique. Les formes asymptomatiques et paucisymptomatiques sont probablement très fréquentes. La proportion élevée d'hospitalisation s'explique probablement par le caractère indéterminé de la fièvre

Aucun lien d'intérêt

\section{TROP-13}

Investigation de cas groupés de cryptosporidiose chez des enfants de la région du haut maroni, Guyane, septembre 2014 à février 2015

N. Martin (1), E. Mosnier (1), L. Favennec (2), G. Roux (1), A. Buteux (1), B. Guarmit (1), L. Epelboin (1), C. Girouin (2), P. Brousse (1), M. Demar (1) (1) $\mathrm{CH}$ de Cayenne,

(2) CHU, Rouen,

(2) ARS de Guyane, Cayenne.

Introduction Entre septembre 2014 et janvier 2015, un nombre anormalement élevé de cas de cryptosporidiose est survenu chez des enfants résidant dans la région du Haut-Maroni. Une investigation a été menée afin de confirmer et décrire cet épisode de cas groupés.

Matériels et méthodes Un cas était défini comme toute personne résidant sur le Haut Maroni ayant eu au moins un examen parasitologique des selles (EPS) positif à Cryptosporidium, entre septembre 2014 et février 2015. Une étude transversale a été menée avec collecte standardisée de données sur les habitudes de vie, la symptomatologie et l'évolution clinique. Une enquête microbiologique avec génotypage et séquençage des souches ainsi qu'un EPS de control à distance de l'épisode aigue a été réalisée. Les données de surveillance climatiques, d'analyses des réseaux d'eau et du fleuve ont étés recueillis au moment et au niveau de la zone d'étude.

Résultats 14 enfants ont été recensés, dont la moitié étaient amérindiens, 6 noirs marrons et 1 franco-brésilien. L'âge médian était de 18 mois, le sexe ratio de 1,8. Les zones de résidence se répartissaient en bord de fleuve, sur tout le HautMaroni. Dans les villages amérindiens, les enfants se baignaient tous dans le fleuve et présentaient les taux d'attaque les plus élevés. Les indicateurs de précarité étaient importants avec pour la majorité des cas $\geq 7$ personnes par foyers et un niveau scolaire de la mère $\leq$ au primaire. La durée des symptômes était de 7,8 jours en moyenne. La morbidité de ces épisodes était élevée : $93 \%$ des patients (13/14) ont présenté une perte de poids avec pour $57 \%$ des cas (8/14) une déshydratation modérée ou sévère. La moitié des enfants (7/14) ont dû bénéficier d'une hospitalisation. Aucun décès n'est survenu. Sept cas contacts étaient retrouvés. Aucun EPS n'était positif à distance (13/14). Le génotypage retrouvai 3 souches différentes à Cryptosporidium hominis en faveur d'une origine multiple du pathogène possiblement environnementale. La même souche: IbA10G2 a cependant été identifié chez trois enfants faisant évoquer un cluster avec une possible part de transmission inter-humaine. La majorité des cas (11/14) sont apparus à l'arrivée de la petite saison des pluies. Les analyses de l'eau de boisson indiquaient des taux trop élevés de turbidité précédemment décrits dans la littérature comme corrélés à l'émergence d'épidémies.

Conclusion Des études prospectives doivent être menées afin de confirmer la nature des facteurs de risques, notamment en ce qui concerne la présence du pathogène dans l'environnement, particulièrement au niveau des bornes d'eau potable et des zones de baignade.

Aucun lien d'intérêt

\section{TROP-14}

Attitudes et pratiques vis à vis du paludisme dans une communauté amérindienne de Guyane

E. Mosnier (1), M. Douine (1), L. Musset (2), S. Rhodes (3), O. Gaudin (1) D. Davy (4), L. Epelboin (1), P. Pieter (5), R. Mutricy (1), F. Djossou (1)

(1) $\mathrm{CH}$ de Cayenne,

(2) Institut Pasteur de la Guyane, Cayenne,

(3) DAAC, Cayenne,

(4) CNRS, Cayenne.

(5) Instituto Oswaldo Cruz, Rio de Janeiro

Introduction Bien que le nombre de cas de paludisme soit en recul en Guyane des foyers touchant les populations autochtones persistent, particulièrement dans certaines zones isolées et frontalières. Afin d'améliorer les stratégies de contrôle du paludisme dans ces zones, nous avons chercher à mieux connaître les attitudes et pratiques vis à vis du paludisme auprès d'une de ces populations-clés.

Matériels et méthodes Une étude transversale a été menée en juin 2015 dans un quartier de Saint Georges de l'Oyapock, frontalier avec le Brésil et présentant une des incidences de paludisme les plus élevées de Guyane (55,2/1 000 habitants en 2013). Les habitants étaient interrogés sur leur attitude en cas d'accès palustre, leurs habitudes de vie et leur mobilité sur ce territoire transfrontalier. Les questionnaires étaient réalisés par des médiateurs en santé issus des communautés interrogées. L'étude a bénéficié d'un accord du Comité de Protection des Personnes.

Résultats Sur 63 habitants du quartier, 57 ont étés inclus dans l'étude. L'âge moyen était de 23,3 ans (mini-max : 2 mois-72 ans). Le sexe ratio homme/femme était de 0,56. Les habitants étaient principalement de nationalité brésilienne (n $=31,53 \%)$ et française $(n=25,43 \%)$. Les langues maternelles parlées étaient essentiellement le karipun $(n=35,60 \%)$, le brésilien $(n=12,21 \%)$ et le paliku $(\mathrm{n}=9,16 \%)$ indépendamment de leur nationalité. Le niveau scolaire était $\leq \mathrm{col}-$ lège pour la majorité des adultes (16/28). Les professions exercées étaient principalement la pêche $(5 / 33)$ et l'agriculture $(6 / 33), 14$ (14/33) personnes étaient sans emploi et 7 avaient d'autres types d'emplois (7/33). Cependant, $13 \%(4 / 31)$ déclaraient avoir une activité liée à l'orpaillage si on leur demandait explicitement. $74 \%(43 / 57)$ se déplaçaient à Oïapoque (ville brésilienne frontalière) au moins une fois par mois. La majoritée se déplaçaient régulièrement en forêt et sur le fleuve sur des zones d'abattis (champs) (32/57), de chasse (31/57) ou de pêche (28/57), sans différence significative selon l'âge ou le sexe. $75 \%(43 / 57)$ déclaraient au moins un antécédent de paludisme dont $12 \%(7 / 43)$ l'année précédan l'enquête. En cas de fièvre, $77 \%$ consultaient un médecin au centre de santé coté français mais $23 \%(13 / 57)$ consultaient uniquement si la fièvre était associée à d'autres symptômes. $46 \%$ (20/43) des personnes rapportaient s'automédiquer.

Conclusion Cette étude retrouve une très forte incidence rapportée de paludisme (127/1 000 habitants en 2014) associée à une fréquence importante d'automédication. Une éducation en santé communautaire en langue maternelle, 
commune aux deux états paraît souhaitable de part et d'autre de la frontière afin d'améliorer la lutte contre le paludisme.

Aucun lien d'intérêt

\section{TROP-15}

Importance du portage asymptomatique de plasmodies dans un quartier à transmission autochtone du paludisme en Guyane : enjeux pour une stratégie d'élimination

E. Mosnier (1), F. Djossou (1), S. Pelleau (2), M. Douine (1), B. Guarmit (1), P. Brousse (1), R. Mutricy (1), C. Simonnet (1), S. Gauduchon (1), L. Musset (2)

(1) $\mathrm{CH}$ de Cayenne,

(2) Institut Pasteur de la Guyane, Cayenne.

Introduction Une fois le contrôle du paludisme atteint, les stratégies d'élimination s'imposent ciblant tout particulièrement les porteurs asymptomatiques de plasmodies. Ces parasitémies asymptomatiques posent des questions scientifiques, éthiques et opérationnelles complexes en terme de détection et de traitement tant sur le plan individuel que collectif. Afin de préciser la part de ces porteurs asymptomatiques en Guyane, nous avons réalisé une étude transversale, en période interépidémique, en population globale, dans un quartier à transmission régulière de paludisme dit autochtone afin d'en connaître la prévalence.

Matériels et méthodes L'étude a été réalisée en juin 2015 dans un quartier de la commune de Saint Georges de l'Oyapock. Toutes les habitants se sont vu proposer un test de diagnostic rapide (TDR) du paludisme et un prélèvement sanguin avec : recherche par PCR de plasmodies et dosage de l'activité enzymatique de la G6PD. Tous les patients ont bénéficiés d'un interrogatoire et d'un examen clinique avec prise de température. Un porteur asymptomatique était défini par une absence d'hyperthermie et de prise d'antipaludiques dans les 15 jours précédant le prélèvement et ayant une PCR positive. Un suivi des cas incidents d'accès aigü a ensuite été réalisé durant les huit mois suivants. Cette étude a bénéficié d'un avis favorable d'un Comité de Protection des Personnes.

Résultats 58 des 63 habitants du quartier ont étés inclus. La moyenne d'âge était de 23,3 ans (min-max : 2 mois-72 ans) et le sexe ratio homme/femme de $0,56.74 \%(43 / 58)$ de la population de l'étude déclarait au moins un antécédent palustre au cours de leur vie dont $12 \%(7 / 58)$ au cours de l'année précédente. Tous les TDR (58/58) étaient négatifs. La PCR a permis d'identifier deux porteurs asymptomatiques : un enfant porteur de $P$. vivax et un adulte porteur de $P$. falciparum. Ceci représentait une prévalence totale de 3,57\% (2/56). Ces deux patients ont étés traités comme pour un accès symptomatique selon les recommandations et n'ont pas développé d'accès palustre symptomatique dans les huit mois qui ont suivi. Cependant, au cours de cette période, trois autres personnes ont développé un accès à $P$. vivax. $3.6 \%(2 / 58)$ des habitants étaient porteur d'un déficit en G6PD. Un enfant et un adulte de langue maternelle amérindienne karipun. Les porteurs asymptomatiques et les cas incidents vivaient tous dans la partie nord du quartier.

Conclusion Cette étude a montré que les porteurs asymptomatiques en dehors des zones hyperendémiques existent. L'intérêt de leur traitement au sein d'une stratégie d'élimination en Guyane reste cependant à objectiver par une étude disposant d'une plus grande puissance.

Aucun lien d'intérêt

\section{TROP-16}

\section{Particularités des formes graves de leptospirose}

C. Tantet (1), L. Collet (1), P. Bourhy (2), M. Jean (1), J. Woessner (1), P. Durasnel (1), L. Valyi (1), S. Henry (3), C. Michaud (1), R. Blondé (1) (1) Centre Hospitalier de Mayotte, Mamoudzou,

(2) Institut Pasteur, Paris,

(3) ARS, Mamoudzou.

Introduction La leptospirose est une maladie de forte incidence en zone tropicale. La présentation clinique ictérohémorragique est la forme grave la plus souvent décrite et volontiers due à Leptospira interrogans sérogroupe icterohaemorrhagiae. Dans notre département, les formes compliquées ne semblent pas présenter les mêmes caractéristiques. L'objectif de ce travail est de décrire les particularités des leptospiroses sévères au sein de notre hôpital.

Matériels et méthodes Cette étude rétrospective monocentrique a été menée de 2009 à 2014. Les formes graves décrites étaient les patients admis en réanimation pour un diagnostic de leptospirose. Ont été exclus les patients dont le diagnostic n'a pas été confirmé bactériologiquement par la technique de Polymerase Chain Reaction (PCR). Une description quantitative des résultats a été réalisée. Les données biologiques correspondent aux valeurs maximales médianes dans les premières 24 heures suivant l'admission.

Résultats Parmi les 612 cas de leptospirose diagnostiqués, 34 formes graves ont été incluses. Le sex ratio est de 5,8 (M/F), l'âge médian de 42 ans. Tous les patients présentent un sepsis sévère ou un choc septique avec un syndrome inflammatoire biologique $(\mathrm{CRP}=303 \mathrm{mg} / \mathrm{L} ; \mathrm{PNN}=9,4 \mathrm{G} / \mathrm{L})$. Près de $60 \%$ (20 patients) sont oliguriques et ont été dialysés; la moitié récupérera une fonction rénale normale, 5 patients garderont des séquelles rénales. L'urémie médiane est de $16,5 \mathrm{mmol} / \mathrm{L}$ et la créatininémie de $242 \mu \mathrm{mol} / \mathrm{L}$. Sur les 12 patients présentant des signes respiratoires, 6 ont été ventilés. Un ictère est présent chez 21 patients avec une bilirubinémie à $122 \mu \mathrm{mol} / \mathrm{L}$, des ASAT à $139 \mathrm{UI} / \mathrm{L}$, des ALAT à $75 \mathrm{UI} /$ $\mathrm{L}$ et une lipase à $275 \mathrm{UI} / \mathrm{L}$. Aucun patient ne présente de signe hémorragique. Tous sont thrombopéniques (plaquettes $=45 \mathrm{G} / 1$ ). Le TP est à $73 \%$. La mortalité s'élève à $8,8 \%$ (3/34). L'identification bactérienne a été possible chez 15 patients : elle permet de mettre en évidence 14 Leptospira sérogroupe Mini et 1 Leptospira sérogroupe Pyrogenes.

Conclusion Les formes graves de leptospirose décrites dans notre département se distinguent par plusieurs particularités : la mortalité est faible, la défaillance respiratoire est au second plan et les formes oligo-anuriques nécessitant une séance de dialyse sont fréquentes. Chez ces patients présentant une défaillance rénale, un suivi est nécessaire pour surveiller la récupération d'une fonction rénale normale. Sur le plan microbiologique, aucun sérogroupe $L$. icterohaemorraghiae n'est présent que c'est celui qui domine dans le département voisin et dans les plupart des zones concernées. En revanche, le sérogroupe Mini est fortement représenté dans notre département qui constitue une singularité bactériologique qui pourrait expliquer des formes graves de leptospiroses différentes de celles dues au sérogroupe icterohaemorrhagiae.

Aucun lien d'intérêt

\section{TROP-17}

La lèpre, une maladie toujours d'actualité dans le $101^{\mathrm{e}}$ département français

D. Mohand-Oussaïd (1), Y. Cazal (1), C. Tabard (1), A. De Montera (1) (1) CHM, Mamoudzou.

Introduction La lèpre reste une maladie à l'état endémique dans ce département : territoire français le plus touché, il affiche l'un des plus forts taux mondiaux de prévalence de la maladie. L'objectif de ce travail est de décrire le dispositif de prise en charge des patients par le Centre Hospitalier de Mayotte (CHM) et de présenter les données disponibles pour les sept dernières années.

Matériels et méthodes Nous avons colligé les dossiers des patients ayant consulté de 2007 à 2014 dans le service de léprologie du CHM. Le diagnostic clinique est systématiquement confirmé par l'histologie. La biopsie cutanée permet de calculer l'index bacillaire (IB), de mieux classifier les patients et de suivre l'évolution de la charge bacillaire au cours du traitement et après. Selon la classification de l'OMS (1988), la biopsie différencie les formes pauci bacillaires (IB $=0$ avec moins de 5 lésions cutanées et atteinte d'un nerf) des formes multibacillaires (IB de $1+$ à 6 avec plus de 5 lésions cutanées et $+/$ - atteinte de plusieurs nerfs). Sur la base des recommandations de l'OMS, la polychimiothérapie associe rifampicine, en dose mensuelle supervisée, en association avec la prise quotidienne de dapsone ou clofazimine pour les formes $\mathrm{PB}$ ou des deux molécules pour les MB. La durée du traitement est de 6 mois pour les PB mais de 24 mois pour les MB.

Résultats De 2007 à 2014, 381 nouveaux cas sont diagnostiqués (vs 342 cas dans une enquête similaire portant sur 1999-2005). Une augmentation des formes MB est observée (35,3\% en 2006 vs $93,86 \%$ en 2014). Le traitement est actuellement bien standardisé. Les complications sont essentiellement de type « réaction de réversion » et érythème noueux lépromateux. Les plus gros foyers concernent 4 communes du nord et du centre de Grande Terre et Petite Terre. Les données 2014 montrent une file active de 154 patients encore en traitement et 54 nouveaux cas, tous confirmés histologiquement (taux de détection de 25,4/100 000 habitants). Parmi les caractéristiques de ces cas incidents : $10,53 \%$ ont moins de 15 ans, plus de la moitié ont un IB élevé ( 4 ou $5+$ ) et $12 \%$ de cas non autochtones et perdus de vue.

Conclusion Cette maladie reste un problème de santé publique à Mayotte (objectif 2005 de l'OMS non atteint, i.e. obtenir une prévalence $<$ à $1 / 10000$ habitants). La forte endémicité régionale et une augmentation des flux migratoires des îles avoisinantes caractérisent la situation. Une possible sous-estimation des cas et la prépondérance des formes conta- 